\title{
Chapter 23 \\ EEG Frontal Asymmetry Related \\ to Pleasantness of Olfactory Stimuli \\ in Young Subjects
}

\author{
Gianluca Di Flumeri, Maria Trinidad Herrero, Arianna Trettel, \\ Patrizia Cherubino, Anton Giulio Maglione, Alfredo Colosimo, \\ Elisabetta Moneta, Marina Peparaio, and Fabio Babiloni
}

\begin{abstract}
It is widely known, in neuroscientific literature, that the brain prefrontal cortex activity asymmetry is closely linked with the pleasantness emotion experienced by the subject during a sensorial stimulation. Thus, from the electroencephalographic (EEG) signal it is possible to estimate the approach/withdrawal index, and this index has been largely investigated and validated in scientific literature, regarding visual and acoustic stimuli. In this work, we present an innovative study aimed to prove, in a systematic way, that such brain AW index is actually correlated with the "pleasant" or "no-pleasant" perception also
\end{abstract}

\section{G. Di Flumeri $(\bowtie) \cdot$ A. Trettel}

BrainSigns srl, Via Sesto Celere, 7c, Rome 00152, Italy

e-mail: gianluca.diflumeri@uniroma1.it; arianna.trettel@brainsigns.com

M.T. Herrero

Clinical and Experimental Neuroscience, Institute of Biomedical Research of Murcia (IMIB),

School of Medicine, University of Murcia, Campus Mare Nostrum, Murcia 30100, Spain

e-mail: mtherrer@um.es

P. Cherubino

Department of Economics and Marketing, IULM University, Milan, Italy

Via Gigi Peronace, Soverato, CZ 88068, Italy

e-mail: patrizia.cherubino@brainsigns.com

A.G. Maglione

BrainSigns srl, Via Sesto Celere, 7c, Rome 00155, Italy

A. Colosimo

Department of Anatomical, Histological, Forensic Medicine and Orthopedic Sciences, Sapienza University of Rome, via Alfonso Borelli, Rome 50-00161, Italy

E. Moneta $\bullet$ M. Peparaio

The Agricultural Research Council - Food and nutrition Research Center (CRA-NUT),

Via Ardeatina, Rome 546-00178, Italy

F. Babiloni

Department of Molecular Medicine, Sapienza University of Rome,

Via di Macchia Palocco 107a, Rome, Italy 
of olfactory stimuli, conveniently produced by standardised methods in the sensory specific scientific literature. In particular, we recorded the electroencephalographic (EEG) signal from a group, gender balanced, of 24 healthy and no-smokers subjects during the perception of ten different smells, presented by means of the "Screening test-odour identification" set (Sniffin' sticks, Burghart). The cerebral AW indexes of all the subjects, for each odorous stimulus, were compared with the appreciation numeric score assessed by the subject during the experiment, by performing a statistical correlation test. Findings show that it is possible to evaluate the pleasantness or no-pleasantness of odorous substances by means of the analysis of EEG signals collected during the presentation of such substances, making way for new applications of such measure kind in experimental environments more and more ecological, as the typical ones of the marketing research areas.

Keywords EEG $\bullet \mathrm{PFC} \cdot$ Alpha asymmetry $\bullet$ Approach-withdrawal $\bullet$ Emotions $\bullet$ Motivations $\bullet$ Pleasantness $\bullet$ Neuromarketing

\subsection{Introduction}

As it is well known in the neuroscientific field, there is a strong relationship between the human brain prefrontal cortex (PFC) activity and motivational processes. In particular, there are evidences about the PFC activity lateralization, with left hemisphere activity increasing for "approach" attitude to emotions, while right hemisphere activity increasing for "withdrawal" attitude. This kind of phenomenon, i.e. the asymmetry of PFC activity, in the scientific literature is called "Approach/Withdrawal motivation" (Davidson 1999), and it has been largely employed as a significant index of positive or negative attitudes of subjects in front of the sensory perception proposed.

By means of electroencephalographic (EEG) techniques, the approach/withdrawal (AW) signal can be estimated as the EEG signal spectral power difference, in the alpha band $(8-12 \mathrm{~Hz}$ ) between the right and left prefrontal cortex (Davidson et al. 1990). Thus, it is possible to estimate in the human such appreciation or avoidance indicators of sensory stimuli by means of some quite simple processing of the EEG signal recorded simultaneously with the proposition of the sensory stimulus.

The AW index is known to be positive in reaction to a sensorial stimulus perceived as "appreciable" by the experimental subject, whilst it will be negative in reaction to a sensorial stimulus perceived as "no appreciable". In particular, this index has been widely investigated and validated in scientific literature, regarding visual (Jackson et al. 2000, 2003; Kong et al. 2013) and acoustic (Schmidt and Trainor 2001; Vecchiato et al. 2012) stimuli.

On the contrary, it has not yet been validated in a systematic way regarding the olfactory stimuli. In fact previous studies just contrasted a couple of smells 
(Kim and Watanuki 2003), whilst it becomes clear that there is the need to validate the brain AW index in reaction to a larger number of olfactory stimuli and in a more controlled experimental conditions.

Hence, this study has the aim to prove in a systematic way that such brain AW index is actually correlated with the "pleasant" or "no-pleasant" perception of olfactory stimuli, conveniently produced by standardised methods in the sensory specific scientific literature.

The results are presented and discussed in the form of a comparison between the AW index values and the experimental subjects evaluation explicit scores, collected by a questionnaire, for the whole proposed smells sample.

\subsection{Materials and Methods}

\subsubsection{Experimental Design}

Twenty-four healthy and nonsmoker subjects, gender balanced $(25 \pm 2.6$ years), were recruited for this study. Informed consent was obtained from each subject after explanation of the study, which was approved by the local institutional ethics committee.

For each subject, his own olfactory sensitivity had previously been established through the validated method "Threshold odour test" (Sniffin' sticks; Heinrich Burghart Elektround Feinmechanik GmbH, Wedel, Germania) (Kobal et al. 1996; Hummel et al. 1997).

The "Sniffin' Sticks" is a test of nasal chemosensory performance based on pen-like odour dispensing devices. It comprises three tests of olfactory function, namely tests for odour threshold, odour discrimination and odour identification. In particular about the odour threshold identification, using a triple-forced-choice paradigm, detection thresholds were determined by employing a single staircase method as described by Doty (1991). The subjects were blindfolded to prevent visual identification of the odorant containing sticks. Three sticks were presented to each subject in a randomised order, two contained only the odourless solvent (propylene glycol in water, $4 \%$ ) and the other the odorant (2-phenylethanol) at a particular dilution in the same solvent. The task of the subject was to indicate the stick with the odorant. Presentation of the triplets to a subject occurred every $20 \mathrm{~s}$, until they had correctly discerned the odorant in two successive trials which triggered a reversal of the staircase. The geometric mean of the last four staircase reversal points of a total of seven reversals was used as the threshold estimate.

By means of this method, the 24 subjects involved in this study were selected within a subjects larger sample. These subjects showed an olfactory sensitivity coherent to the age range considered (2-phenylethanol maximum concentration threshold $=0.063 \%$ ). 


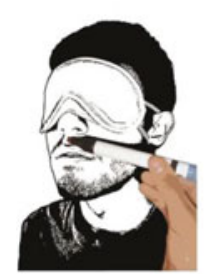

10 seconds

Target odour stimulus

by sniffin' stick

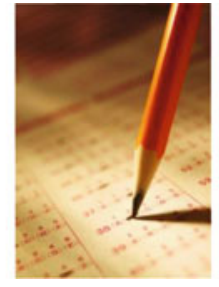

Appreciation judgement $[1 \div 10]$


Milled coffe odor Reset condition

\section{x 10 times \\ 10 odors in a randomized order between the subjects}

Fig. 23.1 Schematic representation of the experimental task described in the paragraph

The experimental task (Fig. 23.1) consisted in presenting to each subject 10 different smells, with an over-threshold concentration, selected within the "Screening test-odour identification" set (Sniffin' sticks, Burghart). In particular, the olfactory stimuli consisted in the following essences: Rose, Banana, Mint, Leather, Cloves, Orange, Pineapple, Fish, Mould, Mushrooms. The odour presentation order was randomised between the subjects in order to prevent the sequence effect as possible confounding effect. The subjects were blindfolded to prevent visual identification of the odorant containing sticks. For each odour presentation, the cap was removed by the experimenter for $\sim 3 \mathrm{~s}$ and the stick tip was placed $\sim 2 \mathrm{~cm}$ in front of both nostrils (Hummel et al. 1997). In the time between the odours presentation the subject was free to open his eyes; in this time interval a questionnaire was submitted to the subject, who had to produce his explicit smell appreciation judgement, with a numeric score between 1 and 10 ( 1 as the worst and 10 as the better judgement in terms of smell appreciation). After the questionnaire and before the following odour presentation, the coffee odour, by means of some milled coffee, was presented to the subject in order to neutralise the previous odour.

\subsubsection{EEG Signal Recording and Processing}

The EEG data was collected, for each subject during the whole experiment, by a portable 64-channel system (BE+ and Galileo software, EBneuro, Italy), at a sampling rate of $256 \mathrm{~Hz}$; impedances kept below $10 \mathrm{k} \Omega$. Nineteen electrodes (Fz, Cz, Pz, Fp1, Fp2, F3, F4, F7, F8, C3, C4, P3, P4, P7, P8, T7, T8, O1, O2) were located on a cap according to the 10-20 international system. FCz was used as reference, $\mathrm{AFz}$ as ground. 
Each EEG trace was then converted into the Brain Vision format (BrainAmp, Brainproducts $\mathrm{GmbH}$, Germany) in order to perform signal pre-processing such as artefacts detection, filtering and segmentation. Thus, raw EEG traces were first band pass filtered (high pass $=2 \mathrm{~Hz}$; low pass $=40 \mathrm{~Hz}$ ), and the Independent Component Analysis (ICA) was then applied to detect and remove components due to eye movements, blinks, and muscular artefacts. Subsequently, the pre-processed EEG signals have been transformed by means of the Common Average Reference (CAR). In the end, for each subject the Individual Alpha Frequency (IAF) was calculated on the 60-s Closed Eyes segment, recorded at the beginning of the experimental task, in order to define the EEG bands of interest according to the method suggested in the current scientific literature, i.e. each band is defined as "IAF $\pm \mathrm{x}$," where IAF is the Individual Alpha Frequency, in Hertz, and $\mathrm{x}$ an integer in the frequency domain (Klimesch 1999).

\subsubsection{Brain Approach/Withdrawal Index}

From the pre-processed EEG data, each channel was filtered in the Alpha band, defined as [IAF-4, IAF+2] according to the IAF definition. Then, the asymmetry of the EEG signal power was evaluated in the Alpha band on the electrodes above the prefrontal and orbitofrontal cortex. The estimation of such asymmetry is called "Approach/Withdrawal (AW) Index". In particular, it is defined as follows:

$\mathrm{AW}=\frac{1}{N_{P}} \sum_{i \in P} x_{\alpha_{i}}^{2}(t)-\frac{1}{N_{Q}} \sum_{i \in Q} y_{\alpha_{i}}^{2}(t)=$ AveragePower $_{\alpha_{\text {right, frontal }}}-$ AveragePower $_{\alpha_{\text {left, frontak }}}$

where $x_{\alpha, i}$ and $y_{\alpha, i}$ are the $i$-th EEG channel in the Alpha band, collected from the frontal right electrodes $P=\{\mathrm{Fp} 2, \mathrm{~F} 4, \mathrm{~F} 8\}$ and the frontal left ones $Q=\{\mathrm{Fp} 1, \mathrm{~F} 3, \mathrm{~F} 7\}$. Thus, for each frontal site (i.e. $P$ and $Q$ ) the Global Field Power (Vecchiato et al. 2012) was estimated, where the $N_{P}$ e $N_{Q}$ values are the cardinality of the two electrodes set. Therefore, the AW index zero value represent an experimental condition where the left and right PFC activity values are equivalent.

For each subject for each olfactory stimulus, the AW signal was calculated. Then, it was averaged along the $10 \mathrm{~s}$ of the stimulation. In conclusion, the correlation value was estimated between the mean AW value and the subjects evaluation explicit score. 


\subsection{Results}

The Fig. 23.2 shows the distribution of appreciation scores attributed by the subjects during the experimentation at the odorous stimuli employed. The vertical bars refer to the appreciation average stated scores obtained by the subjects' whole experimental sample for each odorous substance, indicated along the abscissa axis.

It shows that the fish smell scored the worst evaluation (a mean value of 2.1), on the contrary the Rose, Banana and Mint smells scored the betters. The red line in the graphic refers to the pleasantness threshold, as the total mean score for all the odorous stimuli, equal to 6.1 .

The Fig. 23.3 shows the distribution of the AW index values estimated in the subjects experimental sample during the odorous stimuli presentation. For each odour, the AW value was averaged along the whole subjects sample. On the ordinate axis, the AW scores, where a positive value refers to the approach motivation (i.e. appreciation), whilst a negative value refers to the withdrawal motivation (i.e. rejection) towards the specific odorous stimulus (Davidson et al. 1990).

The Pearson's correlation coefficient between the appreciation stated mean scores and the brain AW mean indexes was estimated, in order to verify the validity of this index in response to olfactory stimuli.

The Fig. 23.4 shows the scatterplot graphic for the ten odorous substances used in this experiment. This graphic type is very useful to highlight the correlation between two variables, in this case the appreciation stated mean scores and the brain AW mean indexes.

\section{Appreciation explicit scores of the odorous stimuli in the subjects} experimental sample

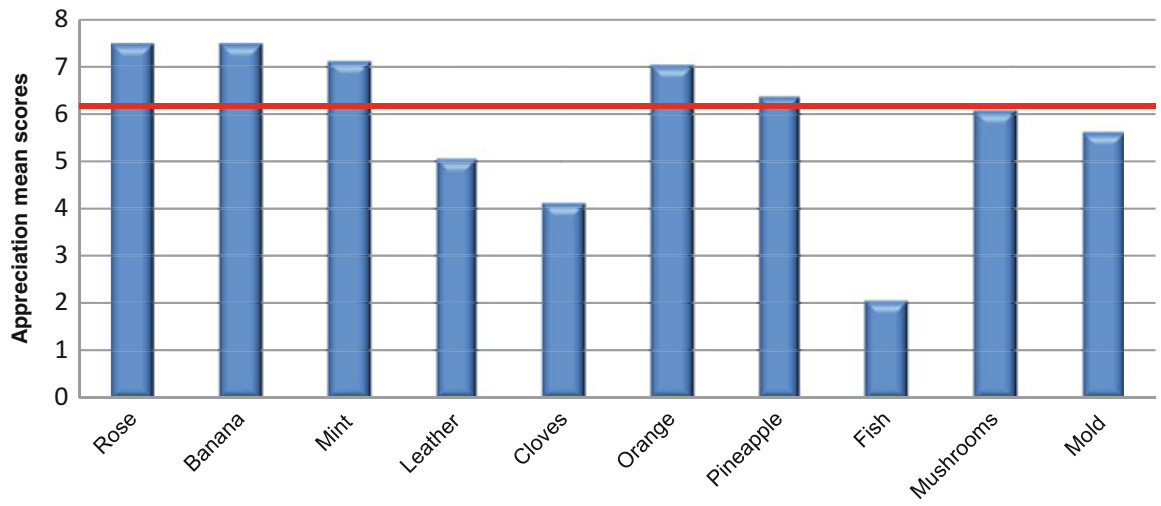

Fig. 23.2 The graphic shows the mean values of explicit appreciation (range between 1 and 10) stated by the subjects experimental sample during the experimentation for each odorous substance. The red line refers to the total mean score, equal to 6.1. It is evident that the Rose, Banana, Mint, Orange and Pineapple smells are above this threshold 


\section{Approach/Withdrawal indexes of the odorous stimuli in the subjects experimental sample}

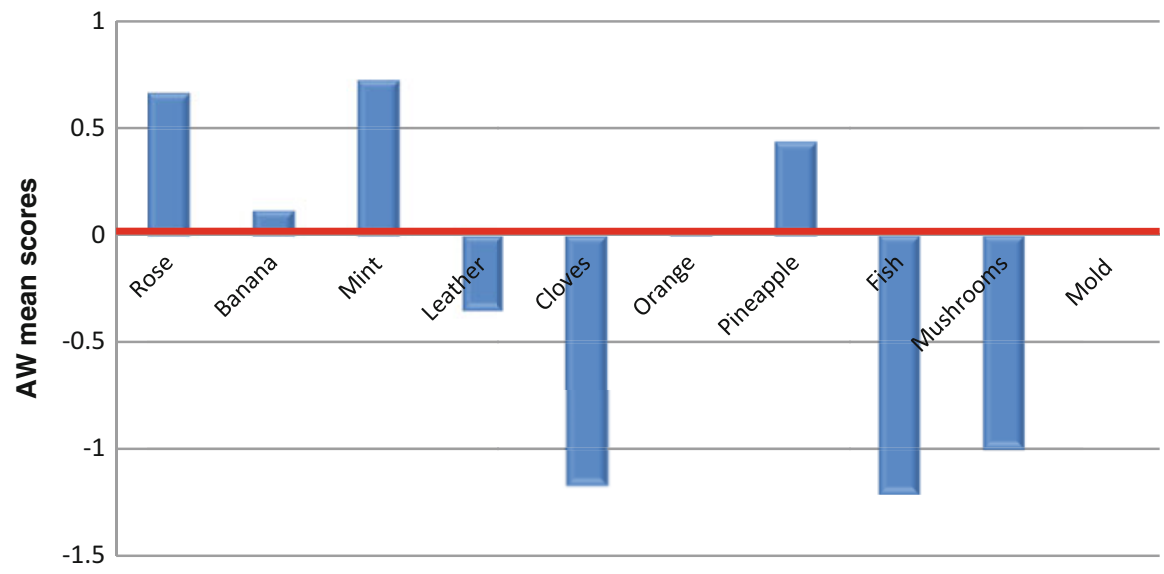

Fig. 23.3 The graphic shows the mean values of the Approach/Withdrawal (AW) index estimated, in the subjects experimental sample, from the electroencephalographic data collected during the odour presentation. The red line corresponds to the AW index zero value, i.e. the indifference situation towards the stimulus. A positive value refers to the approach motivation (i.e. appreciation), whilst a negative value refers to the withdrawal motivation (i.e. rejection) towards the specific odorous stimulus. It is evident that the Rose, Banana, Mint, Pineapple and Mould smells are above this zero level

\section{Correlation between the brain indicator (AW) values and the appreciation stated scores along the smells whole sample $(R=0,79, p<0,0066)$}

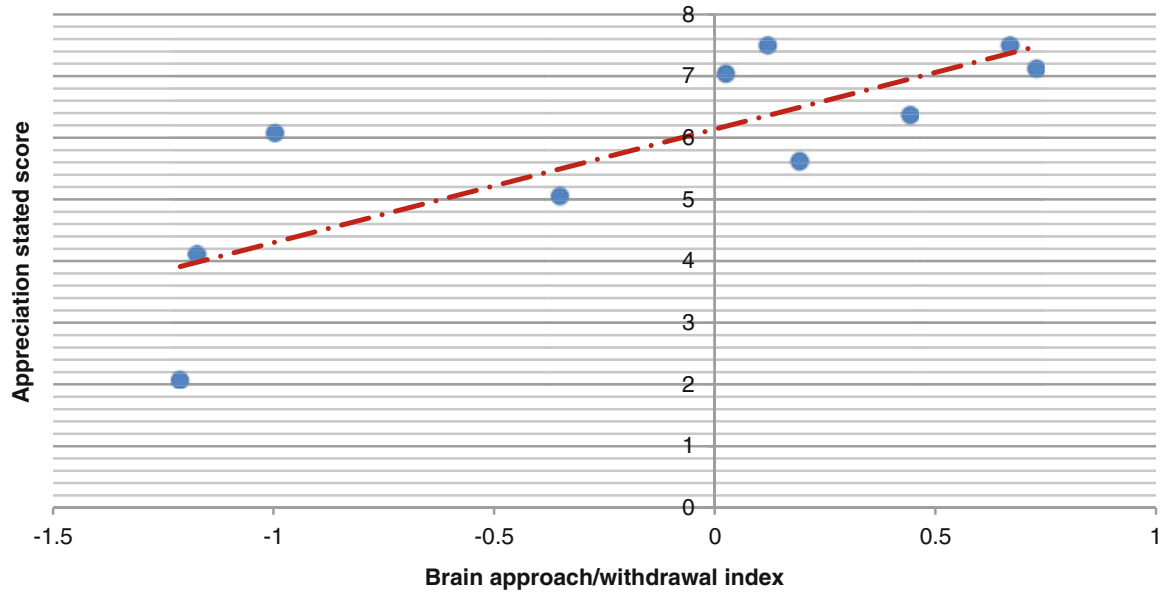

Fig. 23.4 The figure shows the scatterplot between the brain AW index, along the abscissa axis, and the appreciation stated score, between 1 and 10, on the ordinate axis. Each dot in the graphic refers to a specific odorous stimulus used in the experiment. The estimated correlation coefficient $R$ is equal to 0.79 , with a statistical significance of $p<0.006$ 
The estimated Pearson's correlation index $R$ is equal to 0.79 , with a statistical significance index $p$ lower than 0.006 , thus largely inferior to the statistical significance threshold, i.e. $p=0.05$.

\subsection{Discussion}

The positive and significant correlation between the appreciation stated judgement and the appreciation brain indicator clearly proves that the brain approach/withdrawal theory, as indicator of the pleasantness experienced toward a stimulus, continues to be valid also for odorous stimuli, in addition to the widely accepted demonstration with visual and acoustic ones.

Furthermore, by means of the interpolation line in Fig. 23.4 it is possible to appreciate how the brain AW index zero value (indifference towards the smell presented) corresponds approximately to the same threshold level obtained from the appreciation scores assessed by the experimental subjects. In particular, this level is equal to 6.1, is that the threshold to discriminate the pleasant from the no-pleasant stimuli corresponds for both the indicators.

\subsection{Conclusions}

In the present work, the cerebral responses to olfactory stimuli have been studied in a healthy young subjects sample with an olfactory sensitivity coherent to the age range considered, as established by the previous analysis performed. In particular, the results highlight how the brain AW index is an effective indicator for the determination of the appreciation or avoidance motivations towards odorous substances, as an alternative or in support to the appreciation judgements stated by the subjects.

Therefore, it is possible to evaluate the pleasantness or no-pleasantness of odorous substances by means of the analysis of EEG signals collected during the presentation of such substances.

This result seems to be really interesting for those practical applications of these experimental methodologies where it should to be useful to avoid collecting the subjects verbal scores of the olfactory stimulus appreciation, or even worse in such experimental protocols where the real time collection of subjects judgements is impossible.

In addition, another important application of these methodologies could be in the food sciences field, since it should be very useful to better understand how the smells influence the consumers in their food choice. Furthermore, the awareness about the brain motivational processes towards the food could be used to improve the quality of the nutrition, making it more and more pleasant and healthy at the same time. 
In conclusion, the brain activity measure use during the olfactory stimuli perception makes way for new applications of such measure kind in experimental environments more and more ecological.

Acknowledgments The grants provided to Fabio Babiloni by the Italian Ministry of University and Education under the PRIN 2012 and that provided by the Ministry of Foreign Affairs for the bilateral relation between Italy and China for the project "Neuropredictor" are gratefully acknowledged.

\section{References}

Davidson RJ (1999) Neuropsychological perspectives on affective styles and their cognitive consequences. In: Dalgleish T, Power MJ (eds) Handbook of cognition and emotion. Wiley, New York, NY, USA, pp 103-123

Davidson RJ, Ekman P, Saron CD, Senulis JA, Friesen WV (1990) Approach-withdrawal and cerebral asymmetry: emotional expression and brain physiology: I. J Pers Soc Psychol 58 (2):330

Doty RL (1991) Olfactory system. In: Getchell TV, Doty RL, Bartoshuk LM, Snow JB Jr (eds) Smell and taste in health and disease. Raven, New York, p 1803

Hummel T, Sekinger B, Wolf SR, Pauli E, Kobal G (1997) Sniffin' sticks: olfactory performance assessed by the combined testing of odor identification, odor discrimination and olfactory threshold. Chem Senses 22(1):39-52

Jackson DC, Malmstadt JR, Larson CL, Davidson RJ (2000) Suppression and enhancement of responses to emotional pictures. Psychophysiology 37:515-522

Jackson DC, Mueller CJ, Dolski I, Dalton KM, Nitschke JB, Urry HL, Rosenkranz MA, Ryff CD, Singer BH, Davidson RJ (2003) Now you feel it, now you don't frontal brain electrical asymmetry and individual differences in emotion regulation. Psychol Sci 14(6):612-617

Kim YK, Watanuki S (2003) Characteristics of electroencephalographic responses induced by a pleasant and an unpleasant odor. J Physiol Anthropol Appl Human Sci 22(6):285-291

Klimesch W (1999) EEG alpha and theta oscillation reflect cognitive and memory performance: a review and analysis. Brain Res Rev 29(2):169-195

Kobal G, Hummel T, Sekinger B, Barz S, Roscher S, Wolf S (1996) Sniffin' sticks: screening of olfactory performance. Rhinology 34:222-226

Kong W, Zhao X, Hu S, Vecchiato G, Babiloni F (2013) Electronic evaluation for video commercials by impression index. Cogn Neurodyn 7(6):531-535

Schmidt LA, Trainor LJ (2001) Frontal brain electrical activity (EEG) distinguishes valence and intensity of musical emotions. Cognit Emot 15(4):487-500

Vecchiato G, Maglione AG, Scorpecci A, Malerba P, Marsella P, Di Francesco G, Vitiello S, Colosimo A, Babiloni F (2012) EEG frontal asymmetry related to pleasantness of music perception in healthy children and cochlear implanted users. In: Engineering in medicine and biology society (EMBC), 2012 annual international conference of the IEEE, San Diego, CA, pp $4740-4743$ 